\title{
Mobile and Wireless Networks
}

\author{
Ilsun You $^{\mathrm{a}}$ and Takahiro Hara ${ }^{\mathrm{b}}$ \\ ${ }^{a}$ School of Information Science, Korean Bible University, 16 Danghyun 2-gil, Nowon-gu, Seoul, South \\ Kroea \\ E-mail: isyou@bible.ac.kr \\ ${ }^{\mathrm{b}}$ Department of Multimedia Engineering, Graduate School of Information Science and Technology, \\ Osaka University, 1-5 Yamadaoka, Suita, Osaka 565-0871, Japan \\ E-mail: hara@ist.osaka-u.ac.jp
}

Recent advances in wireless and mobile computing technologies have led to a new paradigm of computing by providing a lot of services and functionalities. Wireless technologies allow a wide spectrum of devices including laptops, PDA, intellectual sensors, and cellular phones to exchange and process information. To fulfill the potential for new services, applications and businesses offered by new computing and communication capabilities, several complex research issues need to be further studied and resolved. For example, in order to guarantee the performance requirements from applications in heterogeneous Wireless and Mobile Networks (MWNets), we need to develop new techniques for communication, QoS support, middleware, data management, etc. Also, information exchange among unconstrained entities (e.g. people, server, etc) need security mechanisms to guarantee data confidentiality and integrity and protect services from unauthorized use. This special issue is to address some of these challenges in MWNets.

In response to the call for papers, we received regular papers as well as extended versions of the outstanding papers presented at the third International Conference on Complex, Intelligent and Software Intensive Systems (CISIS-2009) and its workshops. All these submitted papers have gone through a strict review process and among them, six high-quality papers were selected for the publication.

This special issue starts with the paper entitled "Global Consistency Management Methods Based on Escrow Approaches in Mobile Ad Hoc Networks". The authors assume special types of applications in which the instances of each data item can be partitioned and propose two consistency management protocols which are combinations of an escrow method and the authors' previously proposed protocols. The authors also report simulation results to investigate the characteristics of these protocols in a mobile ad hoc network. From the simulation results, it is confirmed that the protocols proposed drastically improve data availability and reduce the traffic for data operations while maintaining the global consistency in the entire network.

The second paper entitled "Using Mobile Devices to Support Online Collaborative Learning", attempts to bridge relevant aspects of mobile technologies in support for collaborative learning and provides a tighter view by means of a multidimensional approach from pedagogical, technological, and evaluation perspectives. Specifically, the authors first analyze the pedagogical perspective in depth, and then study how mobile technologies intersect with the different pedagogical approaches considered. Finally, the 
authors propose an evaluation based on real experiences, which considered the different aspects and perspectives presented.

In the third paper entitled "Architecture for Mobile Heterogeneous Multi Domain Networks", the authors assume Heterogeneous Multi Domain (HMD) networks in which the operations are subject to specific domain policies, and propose a hierarchical architecture, with an infrastructure of gateways at highest control level that enables policy based interconnection, mobility and other services among domains. Besides the architecture, the authors discuss in detail the mobility and adaptive capacity of services in HMD. The authors also discuss the HMD scalability and other advantages compared to existing architectural and mobility solutions. Furthermore, the authors analyze the dynamic availability at the control level of the hierarchy.

The fourth paper entitled "Balanced Bipartite Graph Based Register Allocation for Network Processors in Mobile and Wireless Networks" investigates an approach for efficiently generating balanced bipartite graph and register allocation algorithms for the dual-bank register allocation in Intel's network processors IXPs, which are specially designed for fast packet processing to achieve a broad bandwidth. The paper presents a graph uniform 2-way partition algorithm (FPT), which provides an optimal solution to the graph partition, and a heuristic algorithm for generating balanced bipartite graph. Finally, the authors design a framework for IXP register allocation. Experimental results demonstrate the framework and the algorithms are efficient in register allocation for IXP network processors.

In the fifth paper entitled "The Design and Implementation of Tamper Resistance for Reliable Mobile Game Service", the authors design technical countermeasures for mobile game service forgery and alteration which are the biggest security vulnerabilities deduced from the investigation of status of mobile game service infringement. The proposed "Tamper Resistance Software" architecture consists of Anti Debugging, Anti Disassembling, Anti Memory Dump functions and prevents a reverse analysis and alteration of mobile game service. The outcomes of this study indicate that it is possible to preserve infringements against internal logic of mobile game service by actualizing mobile game planning and development with prudent consideration of adequate countermeasures.

In the last paper entitled "Diffie-Hellman Key Based Authentication in Proxy Mobile IPv6", the authors propose a Diffie-Hellman key based authentication scheme that utilizes the low layer signaling to exchange Diffie-Hellman variables and allows mobility service provisioning entities to exchange mobile node's profile and ongoing sessions securely. The proposed authentication scheme minimizes authentication latency when the mobile node moves across different networks. In addition, pre-established security associations between mobility service provisioning entities are not required so that network scalability in an operationally efficient manner is ensured.

We highly appreciate the effort of all authors in preparing and submitting papers to this special issue. We are very grateful to all reviewers for their time-consuming and meticulous work in judging the papers. We also would like to thank Professor David Taniar, Editor-in-Chief of Mobile Information Systems and IOS Press for helping with the special issue.

Ilsun You received his M.S. and Ph.D. degrees in Computer Science from Dankook University, Seoul, Korea in 1997 and 2002, respectively. From 1997 to 2004, he worked for the THINmultimedia Inc., Internet Security Co., Ltd. and Hanjo Engineering Co., Ltd. as a Research Engineer. Since March 2005, he has been an Assistant Professor in the School of Information Science at the Korean Bible University, South Korea. He served or is currently serving as a general chair or a program chair of international conferences and workshops such as IEEE International Workshop on Intelligent, Mobile and Internet Services in Ubiquitous Computing (IMIS), IEEE International Workshop on Mobile IPv6 and Network-based Localized Mobility Management (MobiWorld), IEEE International Conference on Complex, Intelligent and Software Intensive Systems (CISIS), International Workshop on Managing Insider Security Threats (MIST), International Conference on Broadband and Wireless Computing, Communication and Applications (BWCCA) and so forth. He is in the editorial board for International 
Journal of Ad Hoc and Ubiquitous Computing (IJAHUC), International Journal of Smart Home (IJSH) and Journal of Korean Society for Internet Information (KSII). Also, he served or is currently serving as a guest editor of several journals such as Computing and Informatics (CAI), Wireless Communications and Mobile Computing (WCMC), Intelligent Automation \& Soft Computing (AutoSoft), Journal of Intelligent Manufacturing (JIM), Information Systems Frontiers (ISF), Wireless Personal Communications (WPS), Journal of Universal Computer Science (J.UCS), and Ad Hoc \& Sensor Wireless Networks (AHSWN) and so forth. His main research interests include internet security, authentication, access control, MIPv6 and ubiquitous computing. He is a member of the IEICE, KIISC, KSII, and IEEK.

Takahiro Hara received the B.E, M.E, and Dr.E. degrees from Osaka University, Osaka, Japan, in 1995, 1997, and 2000, respectively. Currently, he is an Associate Professor of the Department of Multimedia Engineering, Osaka University. He has published more than 100 international Journal and conference papers in the areas of databases, mobile computing, peer-to-peer systems, WWW, and wireless networking. He served and is serving as a Program Chair of IEEE International Conference on Mobile Data Management (MDM'06 and 10) and IEEE International Conference on Advanced Information Networking and Applications (AINA'09). He guest edited IEEE Journal on Selected Areas in Communications, Sp. Issues on Peer-to-Peer Communications and Applications. He was a PC Vice-chair of IEEE ICDE'05, IEEE ICPADS'05, IEEE NBiS'09, CSA-09, and IEEE AINA'06, 07, 08, and 10. He served and is serving as PC member of more than 100 international conferences such as IEEE ICNP, WWW, DASFAA, ACM MobiHoc, and ACM SAC. His research interests include distributed databases, peer-to-peer systems, mobile networks, and mobile computing systems. He is an IEEE Senior member and a member of four other learned societies including ACM. 

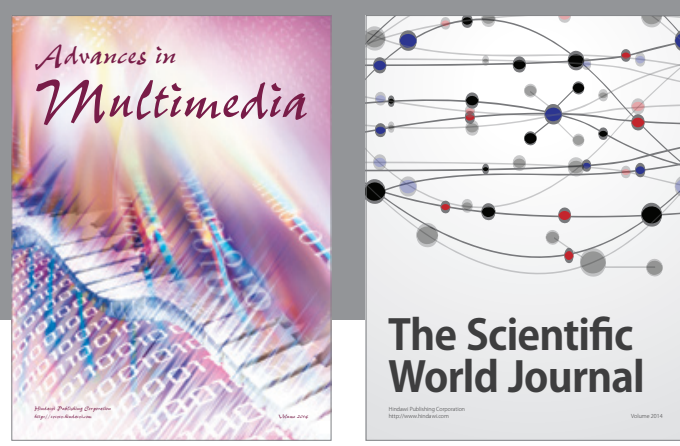

The Scientific World Journal
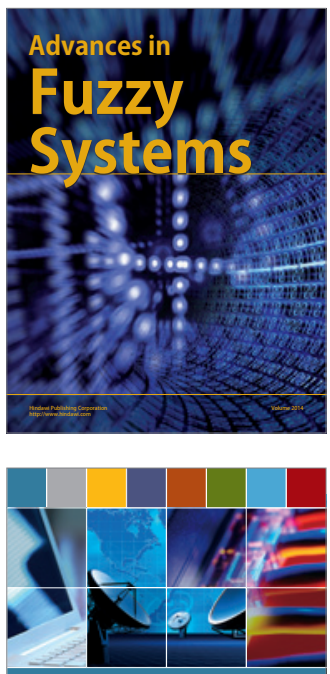

Computer Networks and Communications
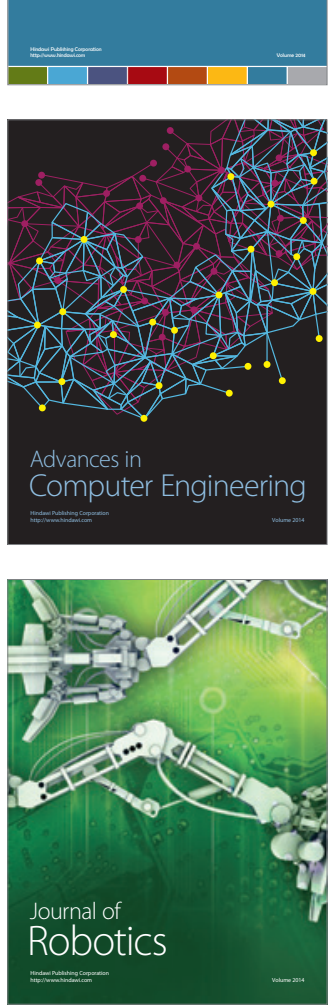
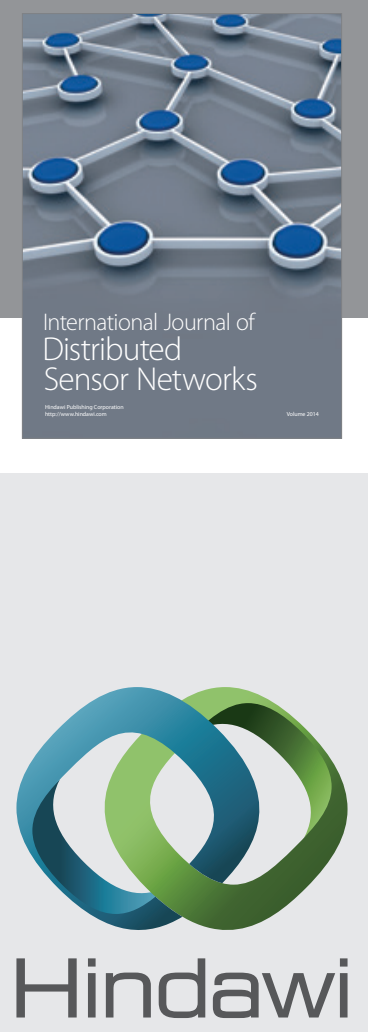

Submit your manuscripts at

http://www.hindawi.com
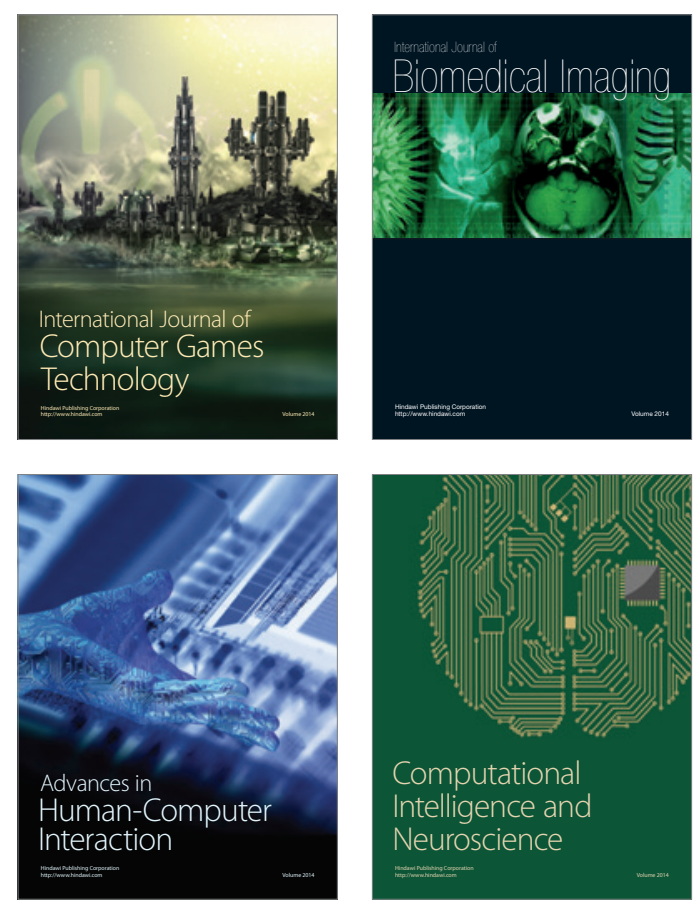
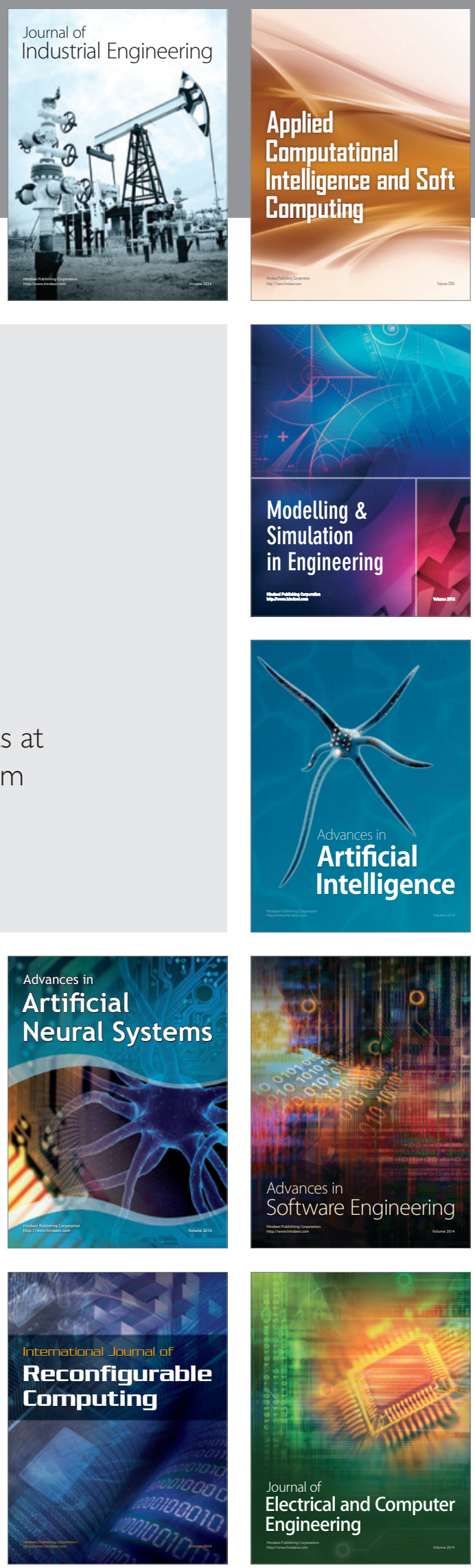